\title{
GEOMETRIC INTERPRETATION OF THE MIXED INVARIANTS OF THE RIEMANN SPINOR
}

\author{
Barry M Haddow* \\ School of Mathematics \\ Trinity College \\ Dublin 2 \\ IRELAND
}

October 9, 2018

\begin{abstract}
Mixed invariants are used to classify the Riemann spinor in the case of Einstein-Maxwell fields and perfect fluids. In the EinsteinMaxwell case these mixed invariants provide information as to the relative orientation of the gravitational and electromagnetic principal null directions. Consideration of the perfect fluid case leads to some results about the behaviour of the Bel-Robinson tensor regarded as a quartic form on unit timelike vectors.
\end{abstract}

*Email:haddow@maths.tcd.ie 


\section{Introduction}

Suppose that $(M, g)$ is a spacetime admitting a spin structure and denote the Weyl and Ricci spinors by $\Psi_{A B C D}$ and $\Phi_{A B C^{\prime} D^{\prime}}$ respectively (the abstract index convention will be used throughout). The most common type of mixed invariant of the Riemann spinor is, roughly speaking, a real or complex scalar constructed from contractions of the Weyl spinor, its complex conjugate and the Ricci spinor, for example the expression $\Psi_{A B C D} \Phi^{A B}{ }_{A^{\prime} B^{\prime}} \Phi^{C D A^{\prime} B^{\prime}}$ defines a mixed invariant. In section two a precise definition of a mixed invariant will be given and their significance will be discussed. The main aim of this paper is to use mixed invariants to provide a classification of the Riemann spinor in the case of spacetimes representing Einstein-Maxwell fields and perfect fluids. This classification will be presented in a fashion which is essentially algorithmic and geometric in character and will provide a geometric interpretation for the mixed invariants. Various aspects of such mixed invariants have been considered by other authors and most recently by Carminati and McLenaghan [1]; McIntosh and Zakhary [2] and Harvey [3] (see also [4]).

Since this paper is only concerned with algebraic properties of the spinors (and tensors) involved it may be assumed that one is working at some particular point of the spacetime manifold. The algebraic classification of the Ricci and Weyl spinors is well known and has been in use in relativity for many years. For details and references see $[5,6]$ (for the Petrov classification of the Weyl spinor/tensor) and [7] (for a review of various methods for classifying the Ricci spinor/tensor). In addition it is possible to effect these classifications in a more or less algorithmic fashion $[8,9,10]$ using pure invariants of the Weyl and Ricci spinors. A pure invariant of the Weyl tensor will be defined in section two but the main ones are, roughly speaking, scalars constructed by contraction of copies of the Weyl spinor, e.g. $\Psi_{A B C D} \Psi^{A B C D}$, and pure invariants of the Ricci spinor can be defined analogously. All these classification schemes must be invariant under $S L(2, \mathbb{C})$ otherwise the schemes would change appearance under a change of dyad.

Despite the large body of literature concerned with the classification of the Ricci and Weyl spinors individually, there has been little or no effort directed to the classification problem for the full Riemann spinor. The complex vector space of Riemann spinor type objects splits as a sum of four irreducible subspaces under the action of $S L(2, \mathbb{C})[11]$ and the components of this decomposition correspond to the Weyl spinor, its complex conjugate, the Ricci spinor and Ricci scalar. Classification of the Riemann spinor in- 
volves the algebraic classification of its irreducible parts, followed by some consideration of the 'angle' between the parts. By this it is meant that the Ricci and Weyl spinors each determine certain directions in spin space and a full classification of the Riemann spinor must take into account the degree of alignment between these directions. Since the Ricci scalar is just a real number and contains no geometrical information it will take no further part in these discussions. Unfortunately different Weyl and Ricci types require separate treatment in the consideration of 'alignment' and this leads to rather a lot of cases to be considered. To reduce the amount of work, only three Ricci types will be studied - those representing null Einstein-Maxwell fields, non-null Einstein-Maxwell fields and perfect fluids. If these three Ricci types are combined with the five possible Petrov types then one arrives at a total of fifteen cases to be considered. These cases are covered in sections 4,5 and 6 where the NPspinor computer algebra package [12] has been used for some of the calculations. It should be noted that all the subcases discussed in these sections may not be realisable in actual spacetimes since restrictions are placed by the Bianchi identities.

As a way of a gentle introduction to the full classification problem, a 'toy problem' will be considered in section three. This toy problem has the benefit of being of some interest in its own right, as well as illustrating some of the important aspects of the Riemann spinor classification. The problem considered in section three is that of the algebraic classification of an ordered pair of symmetric 2 -spinors $\left(\sigma_{A B}, \rho_{A B}\right)$. Since such a pair corresponds to a complex bivector, this problem is relevant to complex general relativity and, in fact, has been studied using other methods by Hall [13]. The classification scheme presented in the third section will involve classifying the individual 2-spinors separately, and then introducing a concept of angle between the pair of 2-spinors.

\section{Mixed Invariants}

The concept of a 'curvature invariant' can be taken to mean a (generally real or complex) scalar constructed from the curvature components which is invariant under the action of an appropriate group of transformations. Such curvature invariants may be appropriate for singularity theory in that singular points may be detected by 'bad' behaviour of these invariants. Other types of curvature invariants may be required in renormalisation theory [14]. 
The approach taken in this work has more in common with the discussion of invariants in the context of algebraic classification given by Penrose and Rindler [5]. The concept of an algebraic invariant discussed in this paper should not be confused with the (related) classical concept of a 'differential concomitant' (see [15] for a modern treatment). It is worth reiterating that throughout this paper it is assumed that one is working at a particular point in the spacetime manifold.

The vector space of unprimed totally symmetric 4-spinors (i.e. Weyl-type spinors) will be denoted by $\mathfrak{W}$ and the vector space of spinors with 2 primed and 2 unprimed indices, which are totally symmetric over both primed and unprimed indices, will be denoted by $\mathfrak{R}$ (corresponding to Ricci-type spinors). The complex conjugate of $\mathfrak{W}$ will simply be denoted by $\mathfrak{W}^{\prime}$. A complex pure invariant of the Weyl spinor (of order $p$ ) is a complex valued multilinear form on $\mathfrak{W}$ which is of $p^{\text {th }}$ order in the Weyl spinor components, and is invariant under the action of $S L(2, \mathbb{C})$ on $\mathfrak{W}$. A real pure invariant of the Weyl spinor can be defined in a similar fashion. The most important pure invariants of the Weyl spinor are traditionally denoted by $I$ and $J$ and are defined by

$$
I=\Psi_{A B C D} \Psi^{A B C D} \quad J=\Psi_{A B}^{C D} \Psi_{C D}{ }^{E F} \Psi_{E F}{ }^{A B}
$$

It is remarked that a pure Weyl invariant, as defined above, need not necessarily be defined by contraction of the Weyl spinor with copies itself, but pure Weyl invariants defined in such a fashion are the only ones required in this work. A pure invariant of the Ricci spinor of order $p$ can be defined in an analogous fashion and the only one required will be the second order real invariant $R_{1}$ defined by

$$
R_{1}=\Phi_{A B A^{\prime} B^{\prime}} \Phi^{A B A^{\prime} B^{\prime}}
$$

A complex basic mixed invariant of order $\left(p, p^{\prime}, q\right)$ is an $S L(2, \mathbb{C})$ invariant multilinear function $M$ defined by

$$
M: \underbrace{\mathfrak{W} \times \cdots \times \mathfrak{W}}_{p \text { times }} \times \underbrace{\mathfrak{W}^{\prime} \times \cdots \times \mathfrak{W}^{\prime}}_{p^{\prime} \text { times }} \times \underbrace{\mathfrak{R} \times \cdots \times \mathfrak{R}}_{q \text { times }} \mapsto \mathbb{C}
$$

where it will be assumed that $\left(p+p^{\prime}\right) q \neq 0$. A real basic mixed invariant can be defined similarly. The appropriate basic mixed invariants which will be required consist of the complex functions

$$
\begin{aligned}
& M_{1}=\Psi_{A B C D} \Phi_{A^{\prime} B^{\prime}}^{A B} \Phi^{C D A^{\prime} B^{\prime}} \\
& M_{2}=\Psi_{A B E F} \Psi_{C D}^{E F} \Phi_{{ }_{A} B^{\prime}}^{A B} \Phi^{C D A^{\prime} B^{\prime}} \\
& M_{5}=\Psi_{A B E F} \Psi_{C D}^{E F} \Phi_{A^{\prime} B^{\prime}} \Phi^{C D}{ }_{C^{\prime} D^{\prime}} \bar{\Psi}^{A^{\prime} B^{\prime} C^{\prime} D^{\prime}}
\end{aligned}
$$


and the real functions

$$
\begin{aligned}
& M_{3}=\Psi_{A B C D} \bar{\Psi}_{A^{\prime} B^{\prime} C^{\prime} D^{\prime}} \Phi^{A B A^{\prime} B^{\prime}} \Phi^{C D C^{\prime} D^{\prime}} \\
& M_{4}=\Psi_{A B E F} \Psi_{C D}^{E F} \bar{\Psi}_{A^{\prime} B^{\prime} E^{\prime} F^{\prime}} \bar{\Psi}^{E^{\prime} F^{\prime}}{ }_{C^{\prime} D^{\prime}} \Phi^{A B A^{\prime} B^{\prime}} \Phi^{C D C^{\prime} D^{\prime}}
\end{aligned}
$$

The notation $M_{1}, \ldots M_{5}$ is as used in [1] except in that reference the authors used a lower case $M$ and a different invariant labelled $M_{4}$. The definition of $M_{4}$ given here is of rather high order, but is required in a few cases to extract certain relevant geometric information. The two real invariants $M_{3}$ and $M_{4}$ can also be expressed in terms of the Bel-Robinson tensor $T_{a b c d}[11]$ and the Ricci tensor $R_{a b}$ according to

$$
\begin{aligned}
& M_{3}=\frac{1}{4} T_{a b c d} R^{a b} R^{c d} \\
& M_{4}=\frac{1}{4} T_{a b e f} T^{e f}{ }_{c d} R^{a b} R^{c d}
\end{aligned}
$$

All the pure invariants and basic mixed invariants may be calculated, in a tensor formalism, using the GRTensor package [16].

Finally, a real or complex mixed invariant is defined as a rational function constructed from pure invariants and basic mixed invariants. It is thus an $S L(2, \mathbb{C})$ invariant rational function of the components of the Weyl spinor, its conjugate and the Ricci spinor.

To close this section, some general remarks concerning the use of invariants, within the context of classification up to some invariance group, will be made. Suppose that a group $G$ acts on a set $S$ and hence decomposes $S$ as a disjoint union of orbits. If $F$ is a $G$-invariant function (taking values in some convenient set - say $\mathbb{R}$ ) then the value of $F$ is constant on each orbit. A collection of $G$-invariant functions $F_{1}, \ldots, F_{p}$ is known as a complete set of invariants if $F_{1}(x)=F_{1}(y), \ldots, F_{p}(x)=F_{p}(y)$ imply that $x$ and $y$ lie on the same orbit. This list of $G$-invariant functions can therefore be used to place each element of $S$ into its appropriate orbit under $G$. As a straightforward example, consider the tangent space $T_{p} M$ under the action of the full Lorentz group $\mathcal{L}$. If the zero vector is excluded then the real-valued function $f(v)=g(v, v)$ provides a complete set of invariants for the tangent space under $\mathcal{L}$. This is because any two vectors of the same size lie in the same $\mathcal{L}$-orbit, and the function $f$ is obviously Lorentz invariant. In this particular case one normally effects a broader classification based on the sign $(+,-$ or 0 ) of $f$ but the invariant $f$ provides the 'finest possible' classification of non-zero vectors in $T_{p} M$ under $\mathcal{L}$. Another method of classifying a non-zero 
tangent vector $v$ is to give its nature (that is: timelike, spacelike or null) together with $|g(v, v)|$ if $v$ is non-null. It is remarked that there is no obvious real-valued Lorentz invariant function on $T_{p} M$ which separates the orbit consisting of null vectors, from the orbit consisting of the zero vector. Instead, one could use the function $g$ which takes the value 1 if the sum of the squares of the components of a vector is non-zero and 0 if this sum vanishes. This function is a perfectly acceptable Lorentz invariant function taking values in the set $\{0,1\}$. Problems analogous to this problem concerning the separation of zero and null vectors also arise in the algebraic classification of the Weyl, Ricci and Riemann spinors using invariants - for example in the separation of Petrov types $N$ and $I I I$ in the algebraic classification of the Weyl tensor [8].

\section{Classification of a Pair of Symmetric 2-Spinors}

For the purposes of this section suppose that $\left(\sigma_{A B}, \rho_{A B}\right)$ is an ordered pair of symmetric 2-spinors which will both be assumed non-zero. As was mentioned earlier, this ordered pair can be identified with a complex bivector $F_{a b}$ by

$$
F_{a b}=\sigma_{A B} \epsilon_{A^{\prime} B^{\prime}}+\bar{\rho}_{A^{\prime} B^{\prime}} \epsilon_{A B}
$$

The classification of a single (non-zero) symmetric 2-spinor is well-known and can be effected by consideration of the pure invariant $I_{1}=\sigma_{A B} \sigma^{A B}$. If $I_{1}=0$ then $\sigma_{A B}$ is said to be null and can be written as $\sigma_{A B}=\alpha_{A} \alpha_{B}$ for some 1-spinor $\alpha_{A}$ (the repeated principal spinor of $\sigma_{A B}$ ) which is defined up to sign. Since all 1-spinors are equivalent under $S L(2, \mathbb{C})$ it follows that all null symmetric 2 -spinors are also equivalent under $S L(2, \mathbb{C})$. If $I_{1} \neq 0$ then $\sigma_{A B}$ is referred to as non-null can be reduced to the canonical form $\mu o_{(A} \iota_{B}$ where $o_{A}, \iota_{A}$ is a normalised spin dyad (that is $o_{A} \iota^{A}=1$ ) and $\mu \in \mathbb{C}$. The dyad is defined up to transformations of the form $o_{A} \mapsto \lambda o_{A}, \iota_{A} \mapsto \lambda^{-1} \iota_{A}$ for $\lambda \in \mathbb{C}$ and the 1 -spinors $o_{A}$ and $\iota_{A}$ are principal spinors of $\sigma_{A B}$. Such a 2-spinor is therefore uniquely determined, up to $S L(2, \mathbb{C})$ transformations, by the complex scalar $\mu$ - given by $-\mu^{2}=2 I_{1}$. The invariant $I_{1}$ does not form a complete set since it takes equal values for $\sigma_{A B}$ and $-\sigma_{A B}$, which lie on different $S L(2, \mathbb{C})$ orbits. It is possible to construct some kind of invariant taking values in $\{-1,1\}$ to separate these two cases but generally such sign problems may be ignored.

Now turn the case of a pair of symmetric 2-spinors $\left(\sigma_{A B}, \rho_{A B}\right)$ and define three invariants in the following manner. Pure invariants $I_{1}$ and $I_{2}$ are defined 
by

$$
I_{1}=\sigma_{A B} \sigma^{A B} \quad I_{2}=\rho_{A B} \rho^{A B}
$$

and a mixed invariant $M$ is defined by

$$
M=\sigma_{A B} \rho^{A B}
$$

In general the set $\left\{I_{1}, I_{2}, M\right\}$ is complete except for the sort of sign problems which arose in the case of a single symmetric 2 -spinor. The cases where this set is not complete are of interest since similar problems arise in the case of the Riemann spinor. For the pair $\left(\sigma_{A B}, \rho_{A B}\right)$ there are really four cases to consider, denoted in an obvious fashion as non-null/non-null, null/non-null, non-null/null and null/null. The middle two cases in this list can clearly be dealt with in the same fashion so one is left with three cases to consider.

Case 1: Non-null/Non-null In this case $I_{1} \neq 0$ and $I_{2} \neq 0$ and it may be assumed that there exist 1-spinors $\alpha_{A}, \beta_{A}, \gamma_{A}$ and $\delta_{A}$ such that

$$
\sigma_{A B}=\alpha_{(A} \beta_{B)} \quad \rho_{A B}=\gamma_{(A} \delta_{B)}
$$

Given four ordered spinor directions, one can define their cross-ratio $[11,17]$ in terms of 1-spinors spanning these directions. The cross-ratio of the four directions spanned by $\alpha_{A}, \beta_{A}, \gamma_{A}$ and $\delta_{A}$ is denoted by $\chi$ and defined by

$$
\chi=\frac{\left(\alpha_{A} \gamma^{A}\right)\left(\beta_{B} \delta^{B}\right)}{\left(\alpha_{C} \delta^{C}\right)\left(\beta_{D} \gamma^{D}\right)}
$$

The function $\chi$ is assumed to take values in $\mathbb{C} \cup\{\infty\}$. If the corresponding flag-pole directions are denoted by $(A, B, C, D)$ then the cross-ratio can be interpreted geometrically in terms of Lorentz transformations required to take the $A-B$ plane into the $C-D$ plane [11]. The cross-ratio $\chi$ of $\alpha_{A}, \beta_{A}, \gamma_{A}$ and $\delta_{A}$ can be expressed in terms of $I_{1}, I_{2}$ and $M$ by

$$
\chi=\frac{\mu+1}{\mu-1} \quad \text { where } \quad \mu^{2}=\frac{M^{2}}{I_{1} I_{2}}
$$

The sign ambiguity in the above definition of $\chi$ corresponds to the arbitrary ordering of $\alpha_{A}$ with respect to $\beta_{A}$ and of $\gamma_{A}$ with respect to $\delta_{A}$. If the directions of two or more of the 1-spinors $\alpha_{A}, \beta_{A}, \gamma_{A}$ and $\delta_{A}$ coincide then 
$\chi$ must take one of the values 0,1 or $\infty$. Since one cannot have $\chi=1$ for finite $\mu$ these degenerate cases correspond to $\mu= \pm 1$ or equivalently $M^{2}=I_{1} I_{2}$. One thus concludes that the condition $M^{2}=I_{1} I_{2}$ is necessary and sufficient for at least one of the principal spinors of $\sigma_{A B}$ to align with one of the principal spinors of $\rho_{A B}$.

The case where $M^{2} \neq I_{1} I_{2}$ is referred to as the non-aligned case and in this case the pair of symmetric 2-spinors is determined (up to sign) by the set of three complex invariants $\left\{I_{1}, I_{2}, M\right\}$. The expression (16) determines the cross-ratio and hence the relative positions of the directions of the four principal spinors up to $S L(2, \mathbb{C})$ rotations [11] and then the invariants $I_{1}$ and $I_{2}$ determine individual scalings.

The aligned $\left(M^{2}=I_{1} I_{2}\right)$ case can be further subdivided into the partially aligned case where exactly one of the principal spinors of $\sigma_{A B}$ is parallel to exactly one of the principal spinors of $\rho_{A B}$; and the totally aligned case where $\sigma_{A B}$ is a multiple of $\rho_{A B}$. In the partially aligned case, if one notes that any three spinor directions are equivalent (under $S L(2, \mathbb{C})$ ) to any other then it is clear that knowledge of $I_{1}$ and $I_{2}$ is sufficient to reconstruct the spinor pair (possibly with some residual sign ambiguity). Similar comments apply in the totally aligned case. The problem is that the invariants $I_{1}, I_{2}$ and $M$ do not appear to be able to separate the partially and totally aligned cases from each other. One method of resolving this problem is to consider the symmetric 2-spinor $\phi_{A B}$ defined by $\phi_{A B}=\sigma_{A C} \rho^{C}{ }_{B}-\rho_{A C} \sigma^{C}{ }_{B}$ and define an invariant $s$ which takes the value 1 if $\phi_{A B} \neq 0$ and 0 if $\phi_{A B}=0$. It may be verified that $s=0$ if and only if $\sigma_{A B}$ is a multiple of $\rho_{A B}$.

then show that $\phi_{A B}=\left(\beta_{C} \gamma^{C}\right) \alpha_{A} \alpha_{B}$

Case 2: Null/Non-null In this case one has that $I_{1}=0$ and $I_{2} \neq 0$ and one can choose a dyad $o_{A}, \iota_{A}\left(\right.$ with $\left.o_{A} \iota^{A}=1\right)$ such that

$$
\left.\sigma_{A B}=\left(o_{A}+B \iota_{A}\right)\left(o_{B}+B \iota_{B}\right) \quad \rho_{A B}=\mu o_{(A} \iota_{B}\right)
$$

for $B, \mu \in \mathbb{C}$. The pair of 2 -spinors are then completely determined, up to $S L(2, \mathbb{C})$ transformations, by the complex quantities $B$ and $\mu$ which are related to the invariants $M$ and $I_{2}$ by $M=-\mu B$ and $I_{2}=-\mu^{2} / 2$. Thus $M$ and $I_{2}$ form a complete set of invariants (apart from a sign ambiguity) and furthermore there is an aligned case corresponding to $M=0$. 


\begin{tabular}{lcccc}
\hline Type & $I_{1}$ & $I_{2}$ & $M$ & $\phi_{A B}$ \\
\hline \hline Non-null/Non-null aligned & $\neq 0$ & $\neq 0$ & $\neq I_{1} I_{2}$ & \\
\hline Non-null/Non-null partially aligned & $\neq 0$ & $\neq 0$ & $=I_{1} I_{2}$ & $\neq 0$ \\
\hline Non-null/Non-null totally aligned & $\neq 0$ & $\neq 0$ & $=I_{1} I_{2}$ & $=0$ \\
\hline Null/Non-null non-aligned & $=0$ & $\neq 0$ & $\neq 0$ & \\
\hline Null/Non-null aligned & $=0$ & $\neq 0$ & $=0$ & \\
\hline Null/Null non-aligned & $=0$ & $=0$ & $\neq 0$ & \\
\hline Null/Null aligned & $=0$ & $=0$ & $=0$ & \\
\hline
\end{tabular}

Table 1: Classification of a pair of 2-spinors. The invariants $I_{1}, I_{2}$ and $M$ are defined by equations (12) and (13) and $\phi_{A B} \equiv \sigma_{A C} \rho^{C}{ }_{B}-\rho_{A C} \sigma^{C}{ }_{B}$

Case 3: Null/Null The final case is characterised by $I_{1}=I_{2}=0$ and the pair of 2-spinors take the form

$$
\sigma_{A B}=\alpha_{A} \alpha_{A} \quad \rho_{A B}=\beta_{A} \beta_{B}
$$

This case can be split into the aligned case ( $\alpha_{A}$ parallel to $\beta_{A}$, equivalent to $M=0)$ and the non-aligned case $(M \neq 0)$. In the non-aligned case, the 2 -spinor pair is determined by the pair of 1 -spinors $\alpha_{A}$ and $\beta_{A}$. However, a pair of non-parallel 1-spinors are determined uniquely (up to $S L(2, \mathbb{C}$ ) transformations) by their inner product and this is given by $M=\left(\alpha_{A} \beta^{A}\right)^{2}$. There is a sign ambiguity in the inner product, but the individual signs of $\alpha_{A}$ and $\beta_{A}$ are irrelevant in the determination of $\sigma_{A B}$ and $\rho_{A B}$. In the nonaligned case, the mixed invariant $M$ is therefore sufficient to determine the pair of 2-spinors up to $S L(2, \mathbb{C})$ transformations. In the aligned case, one has $\rho_{A B}=\lambda \alpha_{A} \alpha_{B}$ for some $\lambda \in \mathbb{C}$. There are clearly no non-zero invariants which can be constructed by contractions of the pair of 2-spinors but different values of $\lambda$ give rise to inequivalent (under $S L(2, \mathbb{C})$ ) pairs of symmetric 2-spinors. With respect to any given dyad the components of $\rho_{A B}$ must be $\lambda$ times the components of $\sigma_{A B}$. Hence one can calculate $\lambda$ by choosing $\mathbf{A}, \mathbf{B}$ such that $\rho_{\mathbf{A B}} \neq 0$ (bold-face indices denote components) and then $\lambda=\rho_{\mathbf{A B}} / \sigma_{\mathbf{A B}}$. The quantity $\lambda$ defined in this way is independent of $\mathbf{A}$ and $\mathbf{B}$ and of the dyad used and $\{\lambda\}$ is a complete set of invariants in the aligned case.

A summary of the discussion in this section is given in table 1 which displays the classification scheme for a pair of non-zero symmetric 2 -spinors using the invariants $I_{1}, I_{2}$ and $M$. 


\section{Null Einstein-Maxwell Fields}

Throughout this section it will be assumed that the Ricci spinor takes the form $\Phi_{A B C^{\prime} D^{\prime}}=\gamma_{A} \gamma_{B} \bar{\gamma}_{C^{\prime}} \bar{\gamma}_{D^{\prime}}$ for some 1-spinor $\gamma_{A}$. Clearly the Ricci spinor is completely determined by $\gamma_{A}$ and in fact $\lambda \gamma_{A}$ will give rise to the same Ricci spinor provided that $|\lambda|=1$. Given that the Ricci spinor is of this form, the necessary mixed invariants required to analyse the relationship between the Weyl and Ricci spinors turn out to be $M_{3}, M_{4}$ and $M_{5}$. The expressions for these invariants are

$$
\begin{aligned}
& M_{3}=\left|\Psi_{A B C D} \gamma^{A} \gamma^{B} \gamma^{C} \gamma^{D}\right|^{2} \\
& M_{4}=\left|\Psi_{A B E F} \Psi_{C D}^{E F} \gamma^{A} \gamma^{B} \gamma^{C} \gamma^{D}\right|^{2} \\
& M_{5}=\left(\Psi_{A B E F} \Psi_{C D}^{E F} \gamma^{A} \gamma^{B} \gamma^{C} \gamma^{D}\right)\left(\bar{\Psi}_{A^{\prime} B^{\prime} C^{\prime} D^{\prime}} \bar{\gamma}^{A^{\prime}} \bar{\gamma}^{B^{\prime}} \bar{\gamma}^{C^{\prime}} \bar{\gamma}^{D^{\prime}}\right)
\end{aligned}
$$

In the above one has that $M_{3} M_{4}=\left|M_{5}\right|^{2}$ and the invariants $M_{3}$ and $M_{5}$ (perhaps together with some pure Weyl invariants) will be seen to form a complete set of invariants for the Riemann spinor in the case of a null Einstein Maxwell field (cf [1]). The invariants $M_{3}$ and $M_{4}$ have a direct geometric interpretation, as will be seen in theorem 1 .

If $\gamma_{A}$ is a repeated principal spinor of the Weyl spinor then the Ricci and Weyl spinors will be said to be repeatedly aligned and similarly the terminology non-repeatedly aligned will refer to the case where $\gamma_{A}$ is a non-repeated principal spinor of the Weyl spinor. It should be noted that Maxwell's equations imply that the flagpole direction of $\gamma_{A}$ is geodesic and shearfree and hence is a repeated principal null direction of the Weyl tensor (by the Goldberg-Sachs theorem [18]) and one is forced into the repeatedly aligned case. In the case of a null fluid one does not have Maxwell's equations and all cases - repeatedly aligned, non-repeatedly aligned and non-aligned may be possible. The following theorem shows how $M_{3}$ and $M_{4}$ may be used to separate these three possibilities.

Theorem 1 Suppose that $\Phi_{A B C^{\prime} D^{\prime}}=\gamma_{A} \gamma_{B} \bar{\gamma}_{C^{\prime}} \bar{\gamma}_{D^{\prime}}$ and that $M_{3}$ and $M_{4}$ are as defined by equations (19) and (20). One has the following three possibilities for alignment between the Weyl and Ricci spinors, assuming both to be nonzero.

(i) Non-aligned $M_{3} \neq 0, M_{4} \neq 0$

(ii) Non-repeatedly aligned $M_{3}=0, M_{4} \neq 0$ 
(ii) Repeatedly aligned $M_{3}=M_{4}=0$

Proof The condition $M_{3}=0$ is clearly equivalent to (repeated or nonrepeated) alignment. If $M_{3}=0$ then let $\eta_{A B}=\Psi_{A B C D} \gamma^{C} \gamma^{D}$ and it follows that $\eta_{A B} \gamma^{A} \gamma^{B}=0$ and $\eta_{A B} \eta^{A B}=0 \Leftrightarrow M_{4}=0$. But then $M_{4}=0$ implies $\eta_{A B} \propto \gamma_{A} \gamma_{B}$ and hence $\eta_{A B} \gamma^{A}=0$. The definition of $\eta_{A B}$ then shows that $\Psi_{A B C D} \gamma^{A} \gamma^{B} \gamma^{C}=0$ i.e. $\gamma^{A}$ is a repeated principal spinor of $\Psi_{A B C D}$. Conversely if $\gamma_{A}$ is a repeated principal spinor of $\Psi_{A B C D}$ it follows that $\eta_{A B} \gamma^{A}=0$ and hence $\eta_{A B}$ is null and so $M_{4}=0$.

For each of the Petrov types the Weyl spinor invariantly determines a normalised dyad $o_{A}, \iota_{A}$ up to some, possibly trivial, group of transformations. The spinor $\gamma_{A}$ can be written in this dyad as $\gamma_{A}=A \iota_{A}-B o_{A}$ so that $A=o_{A} \gamma^{A}$ and $B=\iota_{A} \gamma^{A}$ and, given the indeterminacy in $\gamma_{A}, A$ and $B$ are defined up to transformations of the form $A \rightarrow \lambda A$ and $B \rightarrow \lambda B$ with $\lambda \in \mathbb{C},|\lambda|=1$. For each Petrov type, the appropriate pure Weyl invariants determine the Weyl spinor and then one merely needs to find $A$ and $B$ in order to determine the Ricci spinor and hence the full Riemann spinor. In general $A$ and $B$ can be expressed in terms of the mixed invariants $M_{3}, M_{4}$ and $M_{5}$ and the following is a case by case account of how this is done.

Petrov type $N$ This is arguably the simplest case since both the Ricci spinor and Weyl spinors are each determined by a single 1-spinor. The Weyl spinor can be written as $\Psi_{A B C D}=o_{A} O_{B} O_{C} O_{D}$ for some 1-spinor $o_{A}$, defined up to multiplication by $\lambda \in \mathbb{C}$ satisfying $\lambda^{4}=1$. A dyad $o_{A}, \iota_{A}$ is determined by the Weyl spinor in which the 1-spinor $\iota_{A}$ is arbitrary apart from the normalisation condition $o_{A} \iota^{A}=1$. In the non-aligned case, where $o_{A} \gamma^{A} \neq 0$, the 1-spinor $\iota_{A}$ may be assumed to be parallel $\gamma_{A}$, that is $\gamma_{A}$ can be written as $\gamma_{A}=A \iota_{A}$ for some $A \in \mathbb{R}, A>0$. Under these assumptions one has that $M_{3}=A^{8}$ and hence $M_{3}$ completely determines the relationship between $\gamma_{A}$ and $o_{A}$ and therefore determines the Riemann spinor up to $S L(2, \mathbb{C})$ transformations. In the (necessarily repeatedly) aligned case the Ricci spinor takes the form $\lambda o_{A} o_{B} \bar{o}_{C}^{\prime} \bar{o}_{D}^{\prime}$ for some $\lambda \in \mathbb{R}$. Clearly there are no non-zero mixed invariants which can be formed from contractions of the Weyl and Ricci spinors in this case, and yet different values of $\lambda$ give rise to $S L(2, \mathbb{C})$ inequivalent Riemann spinors. In the aligned case the invariants formed by contractions do not form a complete set and this is reminiscent of the null/null aligned case in section two and one can use similar methods to 
define the invariant $\lambda$.

Petrov type $D$ In this case the Weyl spinor is determined by a pair of spin directions and a complex scalar. One can choose a normalised dyad $o_{A}, \iota_{A}$ spanning the principal directions of the Weyl spinor and determined up to the transformations $o_{A} \rightarrow \lambda o_{A}$ and $\iota_{A} \rightarrow \lambda^{-1} \iota_{A}$ for $\lambda \in \mathbb{C}$. This dyad may be assumed scaled (and reordered if necessary) so that the 1-spinor $\gamma_{A}$ can be written as $\gamma_{A}=A \iota_{A}-o_{A}$ where $A$ is real and non-negative. The Weyl

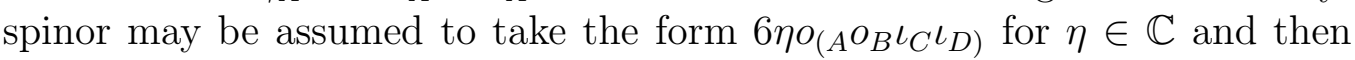
the pure Weyl invariants defined by (1) are given by $I=6 \eta^{2}$ and $J=-6 \eta^{3}$. Now a short calculation will show that $M_{3}=6 A^{4}|I|$ and hence the invariants $M_{3}$ and $I$ will determine the Weyl spinor (up to sign) and the Ricci spinor. The (necessarily repeatedly) aligned case corresponds to $M_{3}=0$ and in this case the only invariant required to determine the Ricci and Weyl spinors (up to sign) is $I$. If one wishes a complete set of invariants, that is if one wishes to remove the sign ambiguity, then one can replace $I$ with $J / I$.

Petrov type $I I I$ In the Petrov type $I I I$ case the Weyl spinor determines (and is determined by) an ordered normalised dyad $o_{A}, \iota_{A}$ subject to the transformation $o_{A} \rightarrow-o_{A}, \iota_{A} \rightarrow-\iota_{A}$. The canonical form in this dyad is $\left.\Psi_{A B C D}=-4 o_{(A} O_{B} O_{C} \iota_{D}\right)$ where $o_{A}$ is a repeated principal spinor and $\iota_{A}$ is a non-repeated principal spinor. Writing $\gamma_{A}=A \iota_{A}-B o_{A}$ as before one has

$$
\begin{aligned}
& M_{3}=\left|4 A^{3} B\right|^{2} \\
& M_{4}=\left|2 A^{4}\right|^{2} \\
& M_{5}=\left(\overline{4 A^{3} B}\right)\left(2 A^{4}\right)
\end{aligned}
$$

It can be confirmed from (23) that $M_{4}=0$ is the necessary and sufficient condition for repeated alignment and that $M_{3} \neq 0$ is equivalent to no alignment. In the non-aligned case and the non-repeatedly aligned case $M_{3}$ and $M_{5}$ determine $4 A^{3} B$ and $2 A^{4}$ up to multiplication by a complex number of unit modulus. Consequently $M_{3}$ and $M_{5}$ determine $A$ and $B$ to within their intrinsic ambiguity and so form a complete set in this case. However, in the repeatedly aligned case $A=0$ and then all three of the mixed invariants $M_{3}$, $M_{4}$ and $M_{5}$ vanish and one has $\Phi_{A B C^{\prime} D^{\prime}}=\lambda o_{A} o_{B} \bar{o}_{C^{\prime}} \bar{o}_{D^{\prime}}$ for some $\lambda \in \mathbb{R}$. It can be seen that there are no mixed non-zero mixed invariants which can be expressed in terms of contractions of the Ricci and Weyl spinors and the 
invariant function $\lambda$ must be determined using similar methods to those used in the null/null aligned case in section two.

Petrov type $I I$ In this case a canonical dyad $o_{A}, \iota_{A}$ is determined to within a finite number of discrete transformations [5] and in this dyad the Weyl spinor takes the form

$$
\left.\Psi_{A B C D}=6 \eta\left(o_{(A} O_{B} \iota_{C} \iota_{D}\right)+o_{A} O_{B} O_{C} O_{D}\right)
$$

The repeated principal spinor in this case is $o_{A}$; the non-repeated principal spinors are $o_{A} \pm i \iota_{A}$ and the pure Weyl invariants are $I=6 \eta^{2}$ and $J=-6 \eta^{3}$. If $\gamma_{A}=A \iota_{A}-B o_{A}$ then the mixed invariants $M_{3}, M_{4}$ and $M_{5}$ are given by

$$
\begin{aligned}
M_{3} & =6|I|\left|P_{1}\right|^{2} \\
M_{4} & =|I|^{2}\left|P_{2}\right|^{2} \\
\bar{I} M_{5} & =-6 I \bar{J} \bar{P}_{1} P_{2}
\end{aligned}
$$

where

$$
P_{1}=A^{4}+B^{2} A^{2} \quad \text { and } \quad P_{2}=2 A^{4}-B^{2} A^{2}
$$

Assuming that $I$ and $J$ are given, equations (26)-(28) provide values for the expressions $\left|P_{1}\right|^{2},\left|P_{2}\right|^{2}$ and $\bar{P}_{1} P_{2}$ and hence determine $P_{1}$ and $P_{2}$ subject to the transformations $\left(P_{1}, P_{2}\right) \rightarrow\left(P_{1} e^{i \theta}, P_{2} e^{i \theta}\right)$ for $\theta \in \mathbb{R}$. However this ambiguity merely reflects the intrinsic ambiguity in $A$ and $B$ and so one can consider the values of the polynomials $A^{4}+B^{2} A^{2}$ and $2 A^{4}-B^{2} A^{2}$ to be completely determined by the mixed invariants. These polynomials will in turn determine the values of $A$ and $B$ except in the case where $A=0$. This exceptional case is the repeatedly aligned case.

In the non-repeatedly aligned case, $A= \pm i B$ and so it may be assumed that $B$ is purely imaginary and that $A$ is real and then $M_{4}=9|I|^{2} A^{8}$ and hence the components of $\gamma_{A}$ in the dyad $o_{A}, \iota_{A}$ are determined by $I$ and $M_{4}$. The case where $M_{4}=0$ and $M_{3} \neq 0$ corresponds to $\gamma_{A}$ being a principal spinor of the square of the Weyl spinor. In the repeatedly aligned case one again finds that all mixed invariants constructed by contractions of the Ricci and Weyl spinors vanish and cannot give a complete classification of the Riemann spinor. 
Petrov type $I$ If the Weyl spinor is of Petrov type $I$ then one possible way of putting it into canonical for is to choose a (unique up to certain discrete transformations [5]) dyad $o_{A}, \iota_{A}$ where $\Psi_{A B C D}$ takes the form

$$
\left.\left.\Psi_{A B C D}=6 \eta\left(\chi O_{(A} \iota_{B} \iota_{C} \iota_{D}\right)+(\chi+1) o_{(A} O_{B} \iota_{C} \iota_{D}\right)+o_{(A} O_{B} O_{C} \iota_{D}\right)
$$

for $\eta, \chi \in \mathbb{C}$. In this case the principal spinors are $o_{A}, \iota_{A}, o_{A}+\iota_{A}$ and $o_{A}+\chi \iota_{A}$ and if one defines $P_{1}$ and $P_{2}$ by

$$
\begin{gathered}
P_{1}=6 \eta A B(A+B)(\chi B+A) \\
P_{2}=-\frac{3}{2} \eta^{2}\left[3 A^{4}+(4+4 \chi) A^{3} B+\left(4+2 \chi+4 \chi^{2}\right) A^{2} B^{2}\right. \\
\left.+\left(4 \chi+4 \chi^{2}\right) B^{3} A+3 \chi^{2} B^{4}\right]
\end{gathered}
$$

then the appropriate pure and mixed invariants are given by

$$
\begin{aligned}
M_{3} & =\left|P_{1}\right|^{2} \\
M_{4} & =\left|P_{2}\right|^{2} \\
M_{5} & =\bar{P}_{1} P_{2} \\
I & =6 \eta^{2}(\chi-\chi+1) \\
J & =-6 \eta^{3}(\chi+1)(\chi-2)\left(\chi-\frac{1}{2}\right)
\end{aligned}
$$

In the non-aligned case $A, B, \eta$ and $\chi$ are determined by $I, J, M_{3}$ and $M_{5}$ as can be shown using similar arguments to those employed in the Petrov type $I I$ case. In the exceptional case where $M_{3}=0$ then $\gamma_{A}$ is parallel to one of the principal spinors of $\Psi_{A B C D}$ and one has either $A=0, B=0, B=-A$ or $B=-A / \chi$ and a complete set of invariants is $I, J$ and $M_{4}$. In these aligned cases $M_{5}=0$ and $M_{4}$ is non-zero and given by the following expressions

$$
\begin{aligned}
A=0, & M_{4}=\left|\frac{9}{2} \chi^{2} \eta^{2} B^{4}\right|^{2} \\
B=0, & M_{4}=\left|\frac{9}{2} \eta^{2} A^{4}\right|^{2} \\
B=-A, & M_{4}=\left|\frac{9}{2} \eta^{2} A^{4}(\chi-1)^{2}\right|^{2} \\
A=-B \chi, & M_{4}=\left|\frac{9}{2} \eta^{2} \chi^{2} B^{4}(\chi-1)^{2}\right|^{2}
\end{aligned}
$$

\section{Non-Null Einstein-Maxwell Fields}

For the purposes of this section it will be assumed that the Ricci spinor can be written as $\Phi_{A B C^{\prime} D^{\prime}}=\phi_{A B} \bar{\phi}_{C^{\prime} D^{\prime}}$ for some non-null symmetric 2-spinor $\phi_{A B}$. 
The Ricci spinor therefore picks out two directions in spin space spanned by $\alpha_{A}$ and $\beta_{A}$, the principal spinors of $\phi_{A B}$. It may be assumed that $\alpha_{A} \beta^{A}=1$ so that $\phi_{A B}=\mu \alpha_{(A} \beta_{B)}$ where the phase of the complex number $\mu$ is arbitrary and so $\mu$ may be chosen real and positive. Also the unordered pair of principal Ricci spinors $\alpha_{A}$ and $\beta_{A}$ are determined subject to the transformations $\alpha_{A} \rightarrow$ $\lambda \alpha_{A}$ and $\beta_{A} \rightarrow \lambda^{-1} \beta_{A}$. The Ricci spinor in this case is completely determined (up to $S L\left(2, \mathbb{C}\right.$ ) transformations) by the real invariant $R_{1}$ which is related to $\mu$ through the equation $4 R_{1}=|\mu|^{4}$. The relevant mixed invariants for this section will be denoted by $N_{1}$ and $N_{2}$ and are defined by $N_{1}=-M_{1} / R_{1}$ and $N_{2}=M_{2} / R_{1}$. These invariants are given by the following equations.

$$
\begin{aligned}
& N_{1}=2 \Psi_{A B C D} \alpha^{A} \beta^{B} \alpha^{C} \beta^{D} \\
& N_{2}=-2 \Psi_{A B E F} \Psi_{C D}^{E F} \alpha^{A} \beta^{B} \alpha^{C} \beta^{D}
\end{aligned}
$$

Petrov type $N$ The Weyl spinor can be written as $\Psi_{A B C D}=o_{A} o_{B} o_{C} o_{D}$ and determines a canonical normalised dyad $o_{A}, \iota_{A}$ in which the direction of $\iota_{A}$ is arbitrary, and $o_{A}$ is subject to multiplication by a fourth root of unity. It may therefore be assumed that $\beta_{A}=\iota_{A}$, by scaling $\beta_{A}$ and/or swapping $\alpha_{A}$ and $\beta_{A}$ as necessary. The condition $\alpha_{A} \beta^{A}=1$ then shows that there exists $A \in \mathbb{C}$ such that $\alpha_{A}=o_{A}+A \iota_{A}$ where, given the ambiguity in $o_{A}$, it may be assumed that $A$ lies in the first quadrant of the complex plane. The mixed invariant $N_{1}$ is then equal to $2 A^{2}$ and hence determines $A$ uniquely and a complete set of invariants for this case is $\left\{R_{1}, N_{1}\right\}$. The special case $A=0$, equivalent to $N_{1}=0$, is the aligned case.

Petrov type $D$ In this case the Weyl spinor and the Ricci spinor each determine a pair of directions in spin space. Since any four ordered directions in spin space are determined by their cross ratio $\chi$ it follows that the Riemann spinor is determined by $\chi, I$ and $R_{1}$ (up to a sign ambiguity). In order to compute the cross ratio, assume that $\left.\Psi_{A B C D}=6 \eta o_{(A} O_{B} \iota_{C} \iota_{D}\right)$, as in the previous section, and suppose that the principal Ricci spinors $\alpha_{A}$ and $\beta_{A}$ are given by

$$
\alpha_{A}=(A B+1) o_{A}+B \iota_{A} \quad \beta_{A}=A o_{A}+\iota_{A}
$$

where $A, B \in \mathbb{C}$. The invariant $N_{1}$ is then given by

$$
N_{1}=2 \eta\left(6 A^{2} B^{2}+6 A B+1\right)
$$


The cross ratio of the four spinors $o_{A}, \iota_{A}, \alpha_{A}, \beta_{A}$ can then be expressed in terms of $A$ and $B$ as follows (recall that $\chi$ takes values in $\mathbb{C} \cup\{\infty\}$ )

$$
\chi=\frac{A B}{A B+1}
$$

A short calculation using (46) and (45) and the expressions for $I$ and $J$ in the type $D$ case, given in the last section, shows that

$$
\chi+\frac{1}{\chi}=\frac{2 N_{1} I-8 J}{N_{1} I+2 J}
$$

Now recall that if any of the four spin directions coincide then the cross ratio must take one of the values 0,1 or $\infty$. From (47) it can be seen that $\chi \neq 1$ for $J \neq 0$ (and $J=0$ is inconsistent with Petrov type $D$ ) and that $\chi=0$ or $\infty$ is equivalent to $N_{1} I+2 J=0$. Thus the aligned case is characterised by the condition $N_{1} I+2 J=0$.

In the non-aligned case, the cross ratio is determined by (47) and the residual ambiguity in the value of $\chi$ corresponds to the ambiguity in the ordering within the pairs $o_{A}, \iota_{A}$ and $\alpha_{A}, \beta_{A}$. The invariants $I, R_{1}$ and $N_{1}$ therefore form a complete set in this case (apart from a sign ambiguity).

The aligned case can be divided into partially aligned, where exactly one of the principal spinors of the Weyl spinor is parallel to a principal Ricci spinor, and totally aligned where both principal spinors of the Weyl spinor are parallel to the Ricci principal spinors. Since any pair of spinor directions is equivalent under $S L(2, \mathbb{C})$ and similarly for any triple of spinor directions it follows that for each type of alignment the appropriate pure invariants form a complete set. This begs the question of how one separates the partially and totally aligned cases and it would appear that there are no suitable real or complex mixed invariants. It is relevant to compare this problem with a similar difficulty which arose during the consideration of the non-null/nonnull aligned case in section 3 . In the present situation the simplest solution seems to be to consider the following expression

$$
\Phi_{A B E^{\prime} F^{\prime}} \Phi^{E^{\prime} F^{\prime} G H} \Psi_{G H C D}-\Phi_{C D E^{\prime} F^{\prime}} \Phi^{E^{\prime} F^{\prime} G H} \Psi_{G H A B}
$$

If one supposes that the Ricci and Weyl spinors are at least partially aligned then one may assume that $\left.\Psi_{A B C D}=6 \eta o_{(A} O_{B} \iota_{C} \iota_{D}\right)$ and $\Phi_{A B C^{\prime} D^{\prime}}=\phi_{A B} \bar{\phi}_{C^{\prime} D^{\prime}}$ where $\phi_{A B}=\mu o_{(A} \beta_{B)}$ and $\beta_{A}=A o_{A}+\iota_{A}$. In this case the expression (48) takes the form

$$
\left.\left.\left.\frac{\mu^{4} \eta}{2}\left[\left(2 o_{(C} \iota_{D}\right)-A o_{C} o_{D}\right) o_{(A} \beta_{B)}-\left(2 o_{(A} \iota_{B}\right)-A o_{A} o_{B}\right) o_{(C} \beta_{D}\right)\right]
$$


The expression (49) clearly vanishes if $\iota_{A}$ is parallel to $\beta_{A}$ (and hence $A=0$ ). Conversely if (49) vanishes then contracting with $\iota^{A} O^{C}$ gives

$$
\frac{3 A \mu^{4} \eta}{4} o_{B} o_{D}=0
$$

which implies that $A=0$. Consequently, in the aligned case the vanishing of (48) is equivalent to total alignment. It is worth remarking that this result also follows if one notes that the vanishing of (48) is equivalent to $\phi_{A B}$ being an eigen-2-spinor of $\Psi_{A B C D}$ and refers to the remarks in [5] concerning the eigen-2-spinor structure of the Weyl spinor.

Petrov type III As in section 4, it may be assumed that $o_{A}$ and $\iota_{A}$ are principal spinors and the Weyl spinor takes the form $\left.\Psi_{A B C D}=-4 o_{(A} O_{B} O_{C} \iota_{D}\right)$. Additionally the Ricci spinor will be assumed to take the form $\mu^{2} \alpha_{(A} \beta_{B)} \bar{\alpha}_{\left(A^{\prime}\right.} \bar{\beta}_{\left.B^{\prime}\right)}$ where $\mu \in \mathbb{R}$ and $\alpha_{A}$ and $\beta_{A}$ are as in (44). The mixed invariants $N_{1}$ and $N_{2}$ are then given by

$$
\begin{aligned}
& N_{1}=4 B(2 A B+1) \\
& N_{2}=4 B^{2}
\end{aligned}
$$

Following [5] a necessary and sufficient condition for alignment is that $Q_{1}=0$ where $Q_{1}$ is defined by

$$
Q_{1}=2 I-4 N_{2}+\left(N_{1}\right)^{2}
$$

In the case of Petrov type $I I I$ one has that $I=0$ and on substituting (51) and (52) into (53) one obtains

$$
Q_{1}=64 B^{3} A(A B+1)
$$

The non-repeatedly partially aligned case is where $A B+1=0$ or $A=0$ but $B \neq 0$ and so $\iota_{A}$ is parallel to $\alpha_{A}$ or $\beta_{A}$ but $o_{A}$ is not parallel to either principal Ricci spinor. Hence from (52), (53) and (54) one can see that necessary and sufficient conditions for non-repeated partial alignment are $N_{2} \neq 0$ and $\left(N_{1}\right)^{2}=4 N_{2}$. In this case $N_{1}$ determines the pair $A, B$ (up to sign) and hence the invariants $N_{1}, R_{1}$ form a complete set.

If $N_{2}=0$ then $N_{1}$ is also zero and $o_{A}$ is parallel to $\alpha_{A}$. Thus, $N_{2}=0$ is a necessary and sufficient condition for repeated partial alignment or total 
alignment. In these cases $\Phi_{A B A^{\prime} B^{\prime}}=\phi_{A B} \bar{\phi}_{A^{\prime} B^{\prime}}$ where $\phi_{A B}=\mu\left(A o_{A} o_{B}+\right.$ $\left.o_{\left(A \iota_{B}\right)}\right)$ and there is no way of determining $A$ from contractions of the Ricci and Weyl spinors. A complete set of invariants is $\left\{A, R_{1}\right\}$ and the partially and totally aligned cases can be separated using similar methods to those used in the type $D$ case.

In the non-aligned case, $A$ and $B$ are determined by $N_{1}$ and $N_{2}$ and so the Riemann spinor is completely fixed by $R_{1}, N_{1}$ and $N_{2}$. The cross ratio $\chi$ of the four spin directions spanned respectively by $o_{A}, \iota_{A}, \alpha_{A}$ and $\beta_{A}$ is given by equation (46) and can be expressed in terms of $N_{1}$ and $N_{2}$ as

$$
\frac{\left(N_{1}\right)^{2}}{4 N_{2}}=\left(\frac{1+\chi}{1-\chi}\right)^{2}
$$

The question of the significance of $N_{1}=0, N_{2} \neq 0$ arises. In this case one can see from (55) that $\chi=-1$ and hence the four principal spinors are harmonic [5]. Equivalently, the flagpole directions of the four spin directions form a square on the celestial sphere of null directions. The other possibilities for the harmonic case are $\chi=2$ or $\chi=\frac{1}{2}$ and these two values for $\chi$ are equivalent to $\left(N_{1}\right)^{2}=36 N_{2}$. In the three harmonic cases one has that $\alpha_{A}$ is parallel to either $A o_{A}+2 \iota_{A}, 2 A o_{A}+\iota_{A}$ and $A o_{A}-\iota_{A}$ where $\beta_{A}$ is as in (44).

Petrov type $I I$ The canonical form for the Petrov type $I I$ Weyl spinor was was given in equation (25) and the dyad $o_{A}, \iota_{A}$ is fixed up to a finite group of transformations. It will be assumed that the Ricci spinor takes the same form as in the preceding discussion of the Petrov type III case.

There are a number of possible types of alignment in the case of a Petrov II non-null Einstein-Maxwell field and the different types will be listed before the interpretation of the mixed invariants is discussed. Firstly, there is the non-aligned case where the set of five spinors consisting of the principal Weyl and Ricci spinors all have different directions in spin-space. In the nonrepeatedly partially aligned case, one of the Ricci principal spinors aligns with one of the non-repeated Weyl principal spinors, and there are no other alignments, whereas in the non-repeatedly totally aligned case the pair of principal Ricci spinors aligns with the pair of non-repeated Weyl spinors. If the repeated principal Weyl spinor aligns with one of principal Ricci spinors and there is no other alignment then the Weyl and Ricci spinors are said to be repeatedly partially aligned. Finally the repeatedly totally aligned case is where the principal spinors of the Ricci spinor are both aligned with principal Weyl spinors - one repeated and one non-repeated. 
In all cases, the Weyl spinor is completely determined (up to sign) by the pure invariant $I$ and then the directions of the Ricci principal spinors are determined by $A$ and $B$. The expressions for $N_{1}$ and $N_{2}$ in terms of $A$ and $B$ are

$$
\begin{aligned}
& N_{1}=-2 \frac{J}{I}\left(6 B\left(B A^{2}+A+B\right)+1\right) \\
& N_{2}=\frac{I}{3}\left(6 B\left(B A^{2}+A-2 B\right)+2\right)
\end{aligned}
$$

In general this pair of equations determine $A$ and $B$ in terms of $N_{1}, N_{2}, J$ and $I$. For example one has

$$
18 B^{2}=1-\frac{3 N_{2}}{I}-\frac{I N_{1}}{2 J}
$$

and then the resulting solution for $B$ can be substituted back to find $A$ (unless $B=0$ ). Noting that $I^{3}=6 J^{2}$, it follows that the set of invariants $I, N_{1}, N_{2}$ and $R_{1}$ determine the Ricci and Weyl spinors, up to sign.

As in the type $I I I$ case, the necessary and sufficient condition for any type of alignment is $Q_{1}=0$ where $Q_{1}$ is defined by (53) and in this case is given by

$$
Q_{1}=24 I B^{2}\left(A^{2}+1\right)\left((A B+1)^{2}+B^{2}\right)
$$

It can be confirmed that $Q_{1}=0$ is equivalent to either $B=0, A= \pm i$ or $A B+1= \pm i B$, which correspond to the different ways of aligning one of the principal Ricci spinors with one of the principal Weyl spinors. In the repeatedly aligned cases (partially or totally) one necessarily has $B=0$ and from (58) one sees that a necessary and sufficient condition for repeated partial or total alignment is $2 I J=6 J N_{2}+I^{2} N_{1}$. In both the repeatedly aligned cases all real and complex mixed invariants formed from contractions can be expressed in terms of the pure invariants and a complete set of invariants is $\left\{I, R_{1}, A\right\}$.

If $B \neq 0$ (equivalently $6 J N_{2}+I^{2} N_{1} \neq 2 I J$ ) but $Q_{1}=0$ then the Ricci spinors must be non-repeatedly aligned - either totally or partially. If one assumes total alignment then it follows that $4 J=I N_{1}$. Conversely if $4 J=$ $I N_{1}$ and $Q_{1}=0$ then it can be shown (from (53)) that $6 N_{2}=7 I$ and equations (56) and (57) imply

$$
\begin{aligned}
B\left(B A^{2}+A+B\right) & =-\frac{1}{2} \\
B\left(B A^{2}+A-2 B\right) & =\frac{1}{4}
\end{aligned}
$$


Solving the above gives $(A, B)=\left( \pm i, \pm \frac{i}{2}\right)$ which is seen to be equivalent to non-repeated total alignment. One therefore has that necessary and sufficient conditions for non-repeated total alignment are $N_{1} I=4 J$ and $6 N_{2}=7 I$. It also follows that $Q_{1}=0, N_{1} I-4 J \neq 0, N_{1} I+2 J \neq 0$ are necessary and sufficient conditions for non-repeated partial alignment (the latter ensuring that $B \neq 0)$. The values of $A$ and $B$ can be determined from $N_{1}$ in the case of partial alignment and so a complete set of invariants (ignoring sign ambiguities) is $\left\{R_{1}, I, N_{1}\right\}$. In the totally aligned case only pure invariants are required to form a complete set.

Petrov type $I$ In this case the Weyl spinor has four non-repeated principal spinors and so there are three possibilities for alignment between the Ricci and Weyl spinors : total, partial or non-aligned. The canonical form for the Weyl spinor is given by (30) and the Ricci spinor will be assumed to take the same form as in the preceding discussion of Petrov types $I I I$ and $I I$. The invariants $N_{1}$ and $N_{2}$ are given by the following rather lengthy expressions.

$$
\begin{aligned}
N_{1} & =-12 \eta \chi A^{3} B^{2}+\left(12(\chi+1) \eta B^{2}-18 \eta \chi B\right) A^{2} \\
& +\left(-12 \eta B^{2}+12(\chi+1) \eta B-6 \eta \chi\right) A \\
& -6 \eta B+2(1+\chi) \eta \\
N_{2} & =9 \eta^{2} \chi^{2} A^{4} B^{2}+\left(-\left(12 \chi^{2}+12 \chi\right) \eta^{2} B^{2}+18 \eta^{2} \chi^{2} B\right) A^{3} \\
& +\left(\left(2+\chi+2 \chi^{2}\right) 6 \eta^{2} B^{2}-18(1+\chi) \chi \eta^{2} B+9 \eta^{2} \chi^{2}\right) A^{2} \\
& +\left(-12(1+\chi) \eta^{2} B^{2}+6\left(2+\chi+2 \chi^{2}\right) \eta^{2} B-6(\chi+1) \chi \eta^{2}\right) A \\
& +9 \eta^{2} B^{2}-6(\chi+1) \eta^{2} B-\left(\chi-4 \chi^{2}-4\right) \eta^{2}
\end{aligned}
$$

From [5] it follows that a necessary and sufficient condition for at least one of the Ricci principal spinors to align with one of the principal Weyl spinors is $Q_{1}=0$ where in this case $Q_{1}$ factorises as follows

$$
Q_{1}=144 \eta^{2} A B(A-1)(\chi A-1)(A B+1)(A B-B+1)(\chi A B-B+\chi)
$$

Noting that the principal Weyl spinors are $o_{A}, \iota_{A}, o_{A}+\iota_{A}$ and $o_{A}+\chi \iota_{A}$, it is clear that the vanishing of $Q_{1}$ is equivalent to at least partial alignment. To determine a condition for total alignment, the easiest method is to assume total alignment and examine the invariants. There are actually nine different ways of aligning the principal Ricci spinors with a pair of principal Weyl 
spinors, but the algebraically simplest possibility would seem to be $A=B=$ 0 . Assuming $A$ and $B$ both zero the pure Weyl invariants are given by (36) and (37) and the mixed invariants are given by

$$
\begin{aligned}
& N_{1}=2 \eta(1+\chi) \\
& N_{2}=\eta^{2}\left(4 \chi^{2}-\chi+4\right)
\end{aligned}
$$

Searching for relationships between $I, J, N_{1}$ and $N_{2}$ one finds that $Q_{2}=0$ where

$$
Q_{2}=6 N_{1} I-3\left(N_{1}\right)^{3}+8 J
$$

Now it must be checked that $Q_{1}=Q_{2}=0$ is necessary and sufficient for total alignment of all types. To achieve this it is necessary to assume partial alignment (i.e. $Q_{1}=0$ ) and, taking each possible solution of $Q_{1}=0$ in turn, factorise the resulting expression for $Q_{2}$, thus solving the equation $Q_{2}=0$. For example, assuming $\beta_{A}$ and $\iota_{A}$ are parallel, that is $A=0$, one has

$$
Q_{2}=648 \eta^{3} B(B-1)(B-\chi)
$$

Thus $Q_{2}=0$ is equivalent to $B=0,1$ or $\chi$ - the conditions for $\alpha_{A}$ to align with one of the other principal Weyl spinors (note that $\eta \neq 0$ for Petrov type $I$ ). The other possible cases can be dealt with similarly and it may be verified that $Q_{1}=Q_{2}=0$ is necessary and sufficient for total alignment.

\section{$6 \quad$ Perfect Fluids}

For the purpose of this section it will be assumed that the trace-free Ricci tensor $S_{a b}$ takes the form

$$
S_{a b}=\frac{1}{4}(\mu+p)\left(4 u_{a} u_{b}-g_{a b}\right)
$$

In the above, $\mu, p \in \mathbb{R}, u_{a}$ is a future pointing unit timelike vector and the spacetime metric $g_{a b}$ has signature -2 . For each of the Petrov types, a canonical dyad $o_{A}, \iota_{A}$ is fixed by the Weyl spinor, up to some subgroup of $S L(2, \mathbb{C})$, and determines a corresponding complex null tetrad $l_{a}, n_{a}, m_{a}, \bar{m}_{a}$ in a standard way [11, page 120]. The complex null tetrad satisfies $l^{a} n_{a}=$ 
$-m^{a} \bar{m}_{a}=1$ with all other inner products zero and thus $u_{a}$ can be expressed in terms of this tetrad according to

$$
u_{a}=A n_{a}+\left(\frac{1+2 C \bar{C}}{2 A}\right) l_{a}+C m_{a}+\bar{C} \bar{m}_{a}
$$

for $C \in \mathbb{C}, A \in \mathbb{R}, A>0$. The components of $\Phi_{A B A^{\prime} B^{\prime}}$ in the corresponding dyad $o_{A}, \iota_{A}$ are then given below. Bold-face indices are used to denote components and the common factor $(\mu+p)$ has been omitted.

$$
\begin{aligned}
& \Phi_{\mathbf{0 0 0}^{\prime} \mathbf{0}^{\prime}}=\frac{1}{2} A^{2} \quad \Phi_{\mathbf{0 1 0 ^ { \prime } \mathbf { 0 } ^ { \prime }}}=-\frac{1}{2} A C \quad \Phi_{110^{\prime} \mathbf{0}^{\prime}}=\frac{1}{2} C^{2} \\
& \Phi_{\mathbf{0 1 0}^{\prime} \mathbf{1}^{\prime}}=\frac{1}{8}+\frac{1}{2} C \bar{C} \quad \Phi_{\mathbf{1 1 0}^{\prime} \mathbf{1}^{\prime}}=-\frac{C(1+2 C \bar{C})}{4 A} \\
& \Phi_{111^{\prime} \mathbf{1}^{\prime}}=\frac{(1+2 C \bar{C})^{2}}{8 A^{2}}
\end{aligned}
$$

The Ricci scalar $R$ has the value $3 p-\mu$ in this case and the other pure Ricci invariant required is $R_{1}$ which haa s the value $\frac{3}{16}(\mu+p)^{2}$ and is necessarily non-zero. The Ricci spinor is uniquely defined, up to sign, by the unit timelike vector $u_{a}$ and the pure invariant $R_{1}$. The role of the mixed invariants in this case is to determine $u_{a}$ in terms of the canonical tetrad fixed by the Weyl tensor. For perfect fluids, appropriate pure invariants are $P_{1}$ and $P_{2}$ and defined by

$$
\begin{aligned}
& P_{1}=\frac{3 M_{3}}{4 R_{1}} \\
& P_{2}=\frac{3 M_{5}}{16 R_{1}}
\end{aligned}
$$

The corresponding tensor expressions for $P_{1}$ and $P_{2}$ are displayed below

$$
\begin{aligned}
& P_{1}=T_{a b c d} u^{a} u^{b} u^{c} u^{d} \\
& P_{2}=\frac{1}{16} \bar{C}^{a g h b}\left(C_{a c d b} C_{g e f h}+\stackrel{*}{C}_{a c d b} \stackrel{*}{C}_{g e f h}\right) u^{c} u^{d} u^{e} u^{f}
\end{aligned}
$$

where $C_{a b c d}$ is the Weyl tensor, $\stackrel{*}{C}_{a b c d}$ its dual and $\bar{C}_{a b c d} \equiv \frac{1}{2}\left(C_{a b c d}+i \stackrel{*}{C}_{a b c d}\right)$ is the anti-self dual part.

It is of interest to discuss the behaviour of $P_{1}$ considered as a quartic form on the space of unit timelike vectors i.e. $P_{1}\left(t^{a}\right)=T_{a b c d} t^{a} t^{b} t^{c} t^{d}$. It 
is known [11] that $P_{1}$ is necessarily positive but one can actually find a lower bound for $P_{1}$ which varies with Petrov type. Before proving a theorem concerning the lower bound of $P_{1}$, some notation will be required. The electric and magnetic parts of the Weyl tensor (with respect to some unit timelike vector $t_{a}$ ) are denoted by $E_{a b}$ and $B_{a b}$ respectively and defined by

$$
E_{a b}=C_{a c d b} t^{c} t^{d} \quad B_{a b}=\stackrel{*}{C}_{a c d b} t^{c} t^{d}
$$

It should be noted that $E_{a b}$ and $B_{a b}$ are both symmetric and orthogonal to $t_{a}$ and are thus elements of the vector space $S=\left\{S_{a b} \mid S_{a b}=S_{b a}, S_{a b} t^{b}=0\right\}$. The vector space $S$ is endowed with a negative-definite inner product defined by total contraction. A duality rotation of angle $\theta$ can be applied to the Weyl tensor $C_{a b c d}$ and transforms it to ${ }^{(\theta)} C_{a b c d}$ where

$$
{ }^{(\theta)} C_{a b c d}=\cos (\theta) C_{a b c d}+\sin (\theta) \stackrel{*}{C}_{a b c d}
$$

The following theorem concerning the lower bound of $P_{1}$ can now be given.

Theorem 2 If $P_{1}\left(t^{a}\right)=T_{a b c d} t^{a} t^{b} t^{c} t^{d}$ then it satisfies the inequality $4 P_{1} \geq|I|$ for all possible unit timelike vectors $t^{a}$. Furthermore, one has $4 P_{1}=|I|$ if and only if there exists $\theta$ such that ${ }^{(\theta)} C_{a b c d} t^{b} t^{c}=0$.

Proof Applying the Cauchy-Schwartz inequality to the inner product space $S$ defined above one obtains

$$
\left(E_{a b} B^{a b}\right)^{2} \leq\left(E_{a b} E^{a b}\right)\left(B_{a b} B^{a b}\right)
$$

with equality if and only if $E_{a b}$ and $B_{a b}$ are linearly dependent. Multiplying each side of (78) by 4 and adding $\left(E_{a b} E^{a b}\right)^{2}+\left(B_{a b} B^{a b}\right)^{2}$ one obtains

$$
\left(E_{a b} E^{a b}-B_{a b} B^{a b}\right)^{2}+4\left(E_{a b} B^{a b}\right)^{2} \leq\left(E_{a b} E^{a b}+B_{a b} B^{a b}\right)^{2}
$$

again with equality if and only if $B_{a b}$ and $E_{a b}$ are parallel. Using the definitions of $E_{a b}$ and $B_{a b}$ to expand the right hand side, one finds that (79) is equivalent to

$$
\left|\left(E_{a b}+i B_{a b}\right)\left(E^{a b}+i B^{a b}\right)\right|^{2} \leq\left(\left(C_{a b c d} C^{a}{ }_{e f}^{d}+\stackrel{*}{C}_{a b c d} \stackrel{*}{a}_{e f}^{d}\right) t^{b} t^{c} t^{e} t^{f}\right)^{2}
$$


Finally it can be seen from [6] that the left hand side of (80) is equal to $|I|^{2}$ (note that the definition of $I$ in that reference differs by a factor of $1 / 2$ from the one used here) and using the definition of $T_{a b c d}$ in terms of the Weyl tensor [11] it can be seen that the right hand side of $(80)$ is equal to $\left(4 P_{1}\right)^{2}$. Consequently the inequality

$$
|I| \leq 4 P_{1}
$$

has been established. This inequality is an equality if and only if $E_{a b}$ and $B_{a b}$ are linearly dependent, that is to say if and only if there exists $\theta$ such that $\cos (\theta) E_{a b}+\sin (\theta) B_{a b}=0$. This last equation can be seen to be equivalent to ${ }^{(\theta)} C_{a b c d} t^{b} t^{c}=0$ and the proof of the theorem is complete.

Defining the complex symmetric tensor $P_{a b} \equiv E_{a b}+i B_{a b}$ and noting that ${ }^{(\theta)} C_{a b c d} t^{b} t^{c}=0$ implies that $E_{a b}$ and $B_{a b}$ are linearly dependent, it follows that $P_{a b}$ is a complex multiple of a real tensor in this case. Hence $P_{a b}$ is diagonable with eigenvalues of equal phase and therefore the Weyl tensor is of Petrov type $I, D$ or $O$. In the Petrov type $I$ case one can see from [5] that the equality of the phases of the eigenvalues implies that the cross-ratio of the four principal spinors is real and hence the Weyl tensor belongs to the subclass of Petrov type $I$ denoted by $I\left(M^{+}\right)$as defined by Arianrhod and McIntosh [19]. It also follows that, in the case of Petrov types $I I, N$ and $I I I$, the lower bound on $P_{1}$ cannot be attained. In fact it will be seen from the calculations which follow that $|I|=4 P_{1}$ would imply that the angle between $t_{a}$ and the (unique) repeated principal null direction vanishes. The value of $4 P_{1}$ therefore approaches $|I|$ as $t_{a}$ approaches the repeated principal null direction.

Petrov type $N$ In this case the freedom in the canonical dyad $o_{A}, \iota_{A}$ determined by writing $\Psi_{A B C D}=o_{A} O_{B} O_{C} O_{D}$ may be used to make $C=0$ in (70). It can then be shown that $P_{1}=A^{4}$, that is to say that $P_{1}=\left(u_{a} l^{a}\right)^{4}$. The value of $A$ is therefore determined by $P_{1}$ and then the Ricci spinor is given by (71) and the pure invariant $R_{1}$. A complete set of invariants for a Petrov type $N$ perfect fluid is therefore $\left\{R, R_{1}, P_{1}\right\}$.

Petrov type $D$ As in the previous two sections the canonical form $\Psi_{A B C D}=$ $\left.6 \eta o_{(A} O_{B} \iota_{C} \iota_{D}\right)$ will be used where the dyad $o_{A}, \iota_{A}$ is defined up to reordering and the rescaling $o_{A} \rightarrow \lambda o_{A}$ and $\iota_{A} \rightarrow \lambda^{-1} \iota_{A}$. The corresponding complex null tetrad is therefore defined up to arbitrary boosts in the $l-n$ plane and 
spatial rotations in the $m-\bar{m}$ plane, which can be used to make $A=1$ and $C=\bar{C}$. In this tetrad the mixed invariant $P_{1}$ is given by

$$
P_{1}=\frac{|I|}{4}\left(1+12 C^{2}+24 C^{4}\right)
$$

The aligned case is where $u_{a}$ lies in the $l-n$ plane and this is equivalent to $C=0$, or $4 P_{1}=|I|$ (the minimum value of $P_{1}$ ). In all cases the direction of $u_{a}$ is determined by $P_{1}$ via (82), which determines $C$. The equation (82) appears to give four possible values for $C$, but since $P_{1}>0$ [11] there is a unique real solution of (82) for $C$ in terms of $P_{1}$ and $|I|$. A complete set of invariants in this case is given by (allowing for sign ambiguities) the pure Ricci invariants $R$ and $R_{1}$, the pure Weyl invariant $I$ and the mixed invariant $P_{1}$.

Petrov type $I I I$ As before the Petrov type $I I I$ Weyl spinor will be assumed to take the canonical form $-4 o_{\left({ }_{A} O_{B} O_{C} \iota_{D}\right)}$ where the dyad $o_{A}, \iota_{A}$ is determined up to a sign change and hence determines a unique complex null tetrad. If $u_{a}$ is given by (70) in this tetrad then the mixed invariants $P_{1}$ and $P_{2}$ are expressed in terms of $A$ and $C$ by the following equations.

$$
\begin{aligned}
& P_{1}=2 A^{2}(1+8 C \bar{C}) \\
& P_{2}=-2 A^{3} \bar{C}
\end{aligned}
$$

Two possible cases arise. The aligned case is where $u_{[a} l_{b} n_{c]}=0$ and is characterised by $C=0$, or equivalently $P_{2}=0$. The non-aligned case corresponds to $P_{2} \neq 0$. In all cases one can clearly determine $C$ and $A$ from $P_{1}$ and $P_{2}$. Consequently a complete set of invariants for the Riemann spinor in this case is $\left\{R, R_{1}, P_{1}, P_{2}\right\}$.

Petrov type $I I$ Assume that the Weyl spinor takes the canonical form (25) where the principal spinors are $o_{A}$ (repeated) and $o_{A} \pm i \iota_{A}$ (non-repeated) in the canonical dyad $o_{A}, \iota_{A}$. There are essentially three cases possible: the repeatedly aligned case where $u_{a}$ lies in the plane spanned by the repeated principal null direction and one of the other principal null directions; the non-repeatedly aligned case where $u_{a}$ lies in the plane spanned by the pair of non-repeated principal null directions; and the non-aligned case. 
The expressions for the invariants $P_{1}$ and $P_{2}$ are

$$
\begin{aligned}
& P_{1}=\frac{3 \eta \bar{\eta}}{2}\left[24 A^{4}+24\left(C^{2}+\bar{C}^{2}\right) A^{2}+\left(24 C^{2} \bar{C}^{2}+12 C \bar{C}+1\right)\right] \\
& P_{2}=\frac{3 \eta^{2} \bar{\eta}}{8}\left[48 A^{4}+24\left(2 \bar{C}^{2}-C^{2}\right) A^{2}-\left(24 C^{2} \bar{C}^{2}+12 C \bar{C}+1\right)\right]
\end{aligned}
$$

Recalling that $I=6 \eta^{2}$ it can be seen that $4 P_{1}$ tends to $|I|$ if $C=0$ and $A$ tends to zero. Notice also that (85) and (86) are equations for $C$ and $A$ in terms of $I / J, P_{1}$ and $P_{2}$. Consideration of $Q_{1} \equiv 4 P_{2}+\eta P_{1}$ (see (87)) enables one to find an expression for $A^{2}$ in terms of $\bar{C}^{2}$ which can be substituted into (86) and the resulting equation split into real and imaginary parts and solved to determine $C$. Thus a complete set of invariants in this case is $\left\{I / J, R, R_{1}, P_{1}, P_{2}\right\}$.

The relationship between the invariants and the various types of alignment will now be examined. The repeated principal null direction is spanned by the vector $l_{a}$ of the canonical tetrad and the non-repeated principal null directions are spanned by $p_{a} \equiv l_{a}+n_{a}-i\left(m_{a}-\bar{m}_{a}\right)$ and $q_{a} \equiv l_{a}+n_{a}+i\left(m_{a}-\bar{m}_{a}\right)$. The necessary and sufficient condition for repeated alignment can be shown to be $A^{2}=-C^{2}$ (which implies that $C$ is purely imaginary). The full expression for $Q_{1}$ defined above is

$$
Q_{1}=108 \eta^{2} \bar{\eta} A^{2}\left(\bar{C}^{2}+A^{2}\right)
$$

and it is clear that $Q_{1}=0$ is equivalent to $A^{2}+C^{2}=0$ (cf [1]) and hence $Q_{1}=0$ is a necessary and sufficient condition for repeated alignment. In the case of non-repeated alignment, one has that $1-2 C^{2}=2 A^{2}$ and $C+\bar{C}=0$ and under these assumptions it is found that $Q_{2} \equiv \eta P_{1}-2 P_{2}-\frac{9}{4} \eta^{2} \bar{\eta}=0$. The expression for $Q_{2}$ in the general case is

$$
Q_{2}=27 \eta^{2} \bar{\eta} C\left(2 C \bar{C}^{2}+2 C A^{2}+\bar{C}\right)
$$

It follows that $Q_{2}=0$ implies that $C$ is purely imaginary and either $2 A^{2}=$ $1-2 C^{2}$ or $C=0$. The former possibility implies non-repeated alignment, but to deal with the $C=0$ case one is required to consider $Q_{3}$ defined by

$$
Q_{3}=7 \eta^{2}\left(P_{1}\right)^{2}-64\left(P_{2}\right)^{2}+\frac{27 \eta^{2} M_{4}}{4 R_{1}}-\frac{189 \eta^{4} \bar{\eta}^{2}}{4}
$$

If $C$ is purely imaginary and $2 A^{2}=1-C^{2}$ then $Q_{3}=0$, but if $C=0$ and $A$ is arbitrary then

$$
Q_{3}=-2916 \eta^{4} \bar{\eta}^{2} A^{4}\left(2 A^{2}-1\right)\left(2 A^{2}+1\right)
$$


Hence $Q_{3}=0$ is equivalent (in the $C=0$ case) to $A= \pm 1 / \sqrt{2}$ which implies $1-2 C^{2}=2 A^{2}$. It has thus been established that necessary and sufficient conditions for non-repeated alignment are $Q_{2}=0=Q_{3}$.

Petrov type $I$ The actual forms of the invariants $P_{1}$ and $P_{2}$ in terms of the components of $u_{a}$ are somewhat complicated and not too illuminating but a result can be proven concerning alignment of the Ricci tensor with the Weyl tensor. The Ricci and Weyl tensors (and spinors) will be said to be aligned when the unit timelike Ricci eigenvalue is parallel to the timelike member of the canonical orthonormal Petrov tetrad [20].

It was mentioned earlier in this section that $P_{1}$ can be considered as a quartic form on unit timelike vectors given by $P_{1}\left(t^{a}\right)=T_{a b c d} t^{a} t^{b} t^{c} t^{d}$. Now define the quadratic form $\gamma\left(t^{a}\right) \equiv g_{a b} t^{a} t^{b}$ and denote the derivatives of $\gamma$ and $P_{1}$ at a point $t^{a}$ in the tangent space by $D_{t} \gamma$ and $D_{t} P_{1}$ respectively. It follows from the theory of Lagrange multipliers [21, page 211] that $t^{a}$ is a critical point of $P_{1}$ restricted to $\gamma^{-1}(1)$ if and only if there exists $\lambda \in \mathbb{R}$ such that $D_{t} P_{1}=\lambda D_{t} \gamma$. It can then be seen that this condition is equivalent to

$$
4 T_{a b c d} t^{b} t^{c} t^{d}=2 \lambda t_{a}
$$

or

$$
t_{[a} T_{b] c d e} t^{c} t^{d} t^{e}=0
$$

It will now be shown that (92) is satisfied with $t_{a}$ replaced by $u_{a}$ if and only if the Ricci and Weyl tensors are aligned. That is to say, it will be shown that (92) is equivalent to $t_{a}$ being the timelike member of the canonical Petrov tetrad (which is unique up to sign). If one defines the self-dual part of the Weyl tensor $\stackrel{+}{C}_{a b c d}$ as $\frac{1}{2}\left(C_{a b c d}-i C_{a b c d}\right)$, so that the anti self-dual part of the Weyl tensor $\left(\bar{C}_{a b c d}\right)$ is the conjugate of the self-dual part, then $T_{a b c d}$ is given by [11]

$$
T_{a b c d}=\stackrel{+}{C}^{p}{ }_{a b}{ }^{q} \bar{C}_{p c d q}
$$

Now suppose that $t_{a}, x_{a}, y_{a}, z_{a}$ is an orthonormal tetrad and define $F_{a b}=$ $2 t_{[a} x_{b]}, G_{a b}=2 t_{[a} y_{b]}$ and $H_{a b}=2 t_{[a} z_{b]}$. If one then defines $\stackrel{+}{F}_{a b}=\frac{1}{2}\left(F_{a b}-i \stackrel{*}{F}_{a b}\right)$, 
$\stackrel{+}{G}_{a b}=\frac{1}{2}\left(G_{a b}-i \stackrel{*}{G}_{a b}\right)$ and $\stackrel{+}{H}_{a b}=\frac{1}{2}\left(H_{a b}-i \stackrel{*}{H}_{a b}\right)$ the self-dual part of the Weyl tensor can be expanded as follows

$$
\begin{aligned}
\stackrel{+}{C}_{a b c d}= & \sigma_{1} \stackrel{+}{F}_{a b} \stackrel{+}{F}_{c d}+\mu_{1}\left(\stackrel{+}{G}_{a b} \stackrel{+}{H}_{c d}+\stackrel{+}{H}_{a b} \stackrel{+}{G}_{c d}\right) \\
+ & \sigma_{2} \stackrel{+}{G}_{a b} \stackrel{+}{G}_{c d}+\mu_{2}\left(\stackrel{+}{H}_{a b} \stackrel{+}{F}_{c d}+\stackrel{+}{F}_{a b} \stackrel{+}{H}_{c d}\right) \\
+ & \stackrel{+}{H}_{a b} \stackrel{+}{H}_{c d}+\mu_{3}\left(\stackrel{+}{F}_{a b} \stackrel{+}{G}_{c d}+\stackrel{+}{G}_{a b} \stackrel{+}{F}_{c d}\right)
\end{aligned}
$$

In the canonical Petrov tetrad one has that $\mu_{1}=\mu_{2}=\mu_{3}=0$ but in a general tetrad $\sigma_{i}$ and $\mu_{i}$ are arbitrary complex numbers, only restricted by $\sigma_{1}+\sigma_{2}+\sigma_{3}=0$. Now recall that $x_{a}, y_{a}$ and $z_{a}$ are arbitrary for a fixed $t_{a}$ (subject to orthogonality relations) and so it may be assumed that they are chosen so that $B_{a b}$ defined by (76) assumes diagonal form. The consequences of this restriction are that $\mu_{1}, \mu_{2}$ and $\mu_{3}$ are real and that the eigenvalues of $B_{a b}$ are -(1/4) times the imaginary parts of $\sigma_{1}, \sigma_{2}$ and $\sigma_{3}$. If the eigenvalues of $B_{a b}$ are distinct then the tetrad $t_{a}, x_{a}, y_{a}, z_{a}$ is now fixed. If there is one pair of equal eigenvalues then it may be assumed, without loss of generality, that $\operatorname{Im}\left(\sigma_{1}-\sigma_{2}\right)=0$ and the eigenvectors with equal eigenvalues are $x_{a}$ and $y_{a}$ and then the further freedom in the tetrad can be used to set $\mu_{3}=0$. The tracefree nature of $B_{a b}$ means that any further eigenvalue degeneracy would force $B_{a b}=0$ and so in this case one can diagonalise $E_{a b}$ and then set $\mu_{1}=\mu_{2}=\mu_{3}=0$.

Using (93) and (94) the condition (92) can be shown to be equivalent to the following three equations

$$
\begin{aligned}
& \mu_{3} \cdot \operatorname{Im}\left(\sigma_{1}-\sigma_{2}\right)=0 \\
& \mu_{2} \cdot \operatorname{Im}\left(\sigma_{3}-\sigma_{1}\right)=0 \\
& \mu_{1} \cdot \operatorname{Im}\left(\sigma_{2}-\sigma_{3}\right)=0
\end{aligned}
$$

Assuming that $B_{a b}$ has no eigenvalue degeneracies the above clearly implies that all the $\mu_{i}$ vanish. If there is an eigenvalue degeneracy of the form discussed earlier, but $B_{a b} \neq 0$, then it may be assumed that $\mu_{3}=0$ and then (96) and (97) imply $\mu_{2}=\mu_{1}=0$. Finally, if $B_{a b}=0$ then all the $\mu_{i}$ automatically vanish. Conversely if all the $\mu_{i}$ vanish then equations (95)(97) are identically satisfied and hence (92) is satisfied. It has therefore been shown that the critical points of $P_{1}\left(t^{a}\right)$ restricted to $\gamma^{-1}(1)$ are merely the two possible timelike members of the canonical Petrov tetrad, which differ only by a sign. Since $P_{1}\left(t^{a}\right)=P_{1}\left(-t^{a}\right)$ it may be assumed that $P_{1}$ is further 
restricted to future pointing members of $\gamma^{-1}(1)$ and it has a unique critical point on this set. It will now be shown that this critical point is the global minimum. Since $\mu_{i}=0$ in the canonical Petrov tetrad (which will be denoted by $v_{a}, x_{a}, y_{a}, z_{a}$ ) one can expand $\stackrel{+}{C}_{a b c d}$ in this tetrad as (writing $\sigma_{i}=\alpha_{i}+i \beta_{i}$ )

$$
\begin{aligned}
\stackrel{+}{C}_{a b c d} & =\left(\alpha_{1} \stackrel{+}{F}_{a b} \stackrel{+}{F}_{c d}+\alpha_{2} \stackrel{+}{G}_{a b} \stackrel{+}{G}_{c d}+\alpha_{3} \stackrel{+}{H}_{a b} \stackrel{+}{H}_{c d}\right) \\
& +i\left(\beta_{1} \stackrel{+}{F}_{a b} \stackrel{+}{F}_{c d}+\beta_{2} \stackrel{+}{G}_{a b} \stackrel{+}{G}_{c d}+\beta_{3} \stackrel{+}{H}_{a b} \stackrel{+}{H}_{c d}\right)
\end{aligned}
$$

where the self-dual bivectors have been redefined in terms of $F_{a b}=2 v_{[a} x_{b]}$, $G_{a b}=2 v_{[a} y_{b]}$ and $H_{a b}=2 v_{[a} z_{b]}$. The decomposition of the self-dual Weyl tensor into the two bracketed quantities above gives rise to a corresponding decomposition of the Weyl tensor $C_{a b c d}=C_{1}{ }_{a b c d}+C_{2} a b c d$. It may then be verified that ${ }^{(\pi / 2)} C_{1} a b c d v^{b} v^{c}=0$ and $C_{2} a b c d v^{b} v^{c}=0$ where ${ }^{(\theta)} C_{a b c d}$ was defined by equation (77). The minimum values of the quartic forms associated with $T_{1} a b c d$ and $T_{2} a b c d$ are shown by theorem 2 to occur at $v^{a}$. Since the following can be shown to hold for any timelike vector $t^{a}$

$$
T_{a b c d} t^{a} t^{b} t^{c} t^{d}=T_{1} a b c d t^{a} t^{b} t^{c} t^{d}+T_{2} a b c d t^{a} t^{b} t^{c} t^{d}
$$

$P_{1}\left(t^{a}\right)$ has a minimum at $v^{a}$ also and the minimum value is given by

$$
P_{1}\left(v^{a}\right)=\frac{1}{16} \sum_{i=1}^{3}\left|\sigma_{i}\right|^{2}
$$

The following theorem has therefore been established

Theorem 3 If the Weyl tensor is of Petrov type $I$ and $u^{a}$ is a future pointing unit timelike vector then the following are equivalent.

(i) $u_{[a} T_{b] c d e} u^{c} u^{d} u^{e}=0$

(ii) $u^{a}$ is the timelike member of the canonical Petrov tetrad.

(iii) $u^{a}$ is the unique global minimum of the quartic form $t^{a} \mapsto T_{a b c d} t^{a} t^{b} t^{c} t^{d}$, restricted to future pointing unit timelike vectors. The minimum value is given by (100).

It is remarked that the condition $u_{[a} T_{b] c d e} u^{c} u^{d} u^{e}=0$ can be expressed in terms of a perfect fluid Ricci tensor to give a necessary and sufficient condition for alignment. 


\section{Acknowledgements}

The author acknowledges the financial support of the European Union in the form of a Human Capital and Mobility post-doctoral fellowship.

\section{References}

[1] J.Carminati and R.G.McLenaghan, J.Math.Phys. 32 (1991) p3135

[2] C.B.G.McIntosh and E.Zakhary in Relativity Today, Proceedings of the Fourth Hungarian Relativity Workshop Edited by R.P.Kerr and Z.Perjés, Akadémiai Kiadó, Budapest (1994) p3

[3] A.Harvey, J.Math.Phys. 36 (1995) p356

[4] R.Penrose, Ann.Physics, 10 (1960) p171

[5] R.Penrose and W.Rindler, Spinors and Space-Time Volume 2 (Cambridge University Press, 1986)

[6] D.Kramer, H.Stephani, M.MacCallum and E.Herlt, Exact Solutions of Einstein's Field Equations (VEB Deutscher Verlag der Wissenschaften, Berlin, 1980)

[7] G.S.Hall in Differential Geometry, Banach Centre Publications Vol.12, p53, (PWN, Polish Scientific Publishers, Warsaw, 1984)

[8] R.A.d'Inverno and R.A.Russell-Clark, J.Math.Phys. 12 (1971) p1258

[9] G.C.Joly and M.A.H.MacCallum, Class.Quant.Grav. 7 (1990) p541

[10] F.W.Letniowski and R.G.McLenaghan, Gen.Rel.Grav. 20 (1988) p463

[11] R.Penrose and W.Rindler, Spinors and Space-Time Volume 1 (Cambridge University Press, 1984)

[12] S.R.Czapor, R.G.McLenaghan and J.Carminati, Gen.Rel.Grav. 24 (1992) p911

[13] G.S.Hall, Acta.Phys.Pol. B 17 (1986) p139 
[14] S.A.Fulling, R.C.King, B.G.Wybourne and C.J.Cummins, Class.Quant.Grav. 9 (1992) p1151

[15] J.M.Masqué and A.Valdés, To appear in J.Phys A.

[16] D.Pollney, The GRTensor Resource Home Page, URL http://astro.queensu.ca/ grtensor/GRHome.html

[17] J.G.Semple and G.T.Kneebone, Algebraic Projective Geometry (Oxford University Press, 1952)

[18] J.N.Goldberg and R.K.Sachs, Act.Phys.Pol. Suppl. 22 (1962) p13

[19] R.Arianrhod and C.B.G.McIntosh, Class.Quant.Grav. 9 (1992) p1969

[20] J.Ehlers and W.Kundt in Gravitation: An Introduction to Current Research edited by L.Witten, (Wiley, New York, 1962)

[21] R.Abraham, J.E.Marsden and T.Ratiu, Manifolds, Tensor Analysis, and Applications (Springer-Verlag, New York, 1988) 